personal ornament, distinctive of the Early Bronze Age of Central Europe, have been multiplied surprisingly during the last five years. Ingot-torques, lockrings and ear-rings with flattened ends, scroll, knot-headed and racquet pins are now all known from Mesopotamia.

(4) In Mesopotamia, however, all these types emerge unexpectedly early. The terminus post quem for the beginning of the Central European Bronze Age or Danubian IV is accordingly nearer 2800 than 1800 B.c. Moreover, types distinctive of previous phases of European prehistory have come to light in still earlier contexts in the Orient ; battle-axes, for example, such as belong to Danubian III, can be traced back to the Uruk or even the al'Ubaid phase in Mesopotamia. Still adhering to a strict application of axiom (4), we can raise the absolute dates attributed to the several phases of the New Stone Age in Europe to meet the estimates of botanists and geologists.

(5) Even so, axiom (5) would remain intact. At the beginning of the Central European Bronze Age about 2800 B.c., we should survey a cultural continuum over which we descend by regular gradations from the great cities of Egypt and Mesopotamia with their fully literate civilization through Anatolian and Agean townships of illiterate, but metal-using communities, to Central European villages where metal was virtually restricted to weapons and ornaments, neolithic hamlets in North Germany and Denmark and pure food-gatherers farther north. At the beginning of the European neolithic period, a couple of millennia earlier, similar relations would hold good: a chalcolithic Assyrian township like Arpachiya would have to be compared to a self-sufficing temporary hamlet of twenty households like Köln-Lindenthal, while the boundaries of foodgatherers would be extended southward to the edge of the loess in South and Central Germany.

Even on such a maximal chronology, the orientalist position remains unassailable. But that chronology has been advanced only tentatively to see how archæological deductions fit the hypotheses of other disciplines. It need not be accepted as even plausible unless geologists and botanists become more unanimous and confident.

\section{Eye and Brain as Factors in Visual Perception}

$\mathrm{N}$ his presidential address to Section $\mathrm{J}$ (Psychology), Dr. R. H. Thouless deals with the implications for the theory of visual perception of the fact that the physiological mechanism of vision is not only the retinal surface but also the whole system which includes retina, optic nerve, visual area of the cortex, and to some extent, other sensory areas of the brain as well.

It was assumed in much of the earlier work in vision (as in that of Helmholtz) that the basic process in vision is the formation of an optical image on the retina and its transmission to the visual centres of the brain by means of the optic nerve. Differences between the sensations transmitted to the brain and the finished perception were attributed to the action of the higher processes of judgment and the influence of past experience. This may be called the 'transmission theory' of vision. Within a certain limited fieldthat of the sensory physiology of the retina-it proved itself a fruitful guide to research. Modern researches on visual perception have, however, now made it clear that the transmission theory is wrong, and that a wholly different way of approaching the problems of visual perception is necessary if we are not to be led astray.

The modern attack on the transmission theory started from Wertheimer's experiments on the socalled 'phi-movement' which is seen when two retinal points are successively stimulated at certain time intervals. This is one of many known examples of the appearance in perception of something which does not exist in the pattern of stimulation.

The same conclusions may be reached by a study of the apparent shape of an inclined object. This apparent shape is not identical with the retinal shape but lies between this and the real shape. Careful consideration of this experiment makes untenable the view that the retinal shape is given as 'sensation', which is modified by a process of 'perceptual judgment'. There is no evidence that the 'sensa. tion' is in any way an ingredient of the experienced shape. Rather the experienced shape appears to be a direct product of both the shape of retinal stimulation and of such perceptual cues as indicate the real shape of the object.

Individual differences are characteristic of the cerebral side of perception. Although the optical system of the eye may not differ much in different individuals, their perceptual responses may be very different. The amount of "phenomenal regression to the 'real' characters of objects" (that is, the extent to which apparent shape, size, and brightness are determined by the real shapes, sizes and brightnesses of objects, irrespective of inclination, distance, and illumination respectively) differs widely from one individual to another. These differences are not generally suspected until they are shown by experiment. The measurements of any one individual, on the other hand, show high self-consistency, even when the period between two measurements is as long as two years.

These differences are not merely of interest to 
scientific curiosity; they may also have practical consequences. Research has confirmed the prediction (originally made on theoretical grounds) that a high tendency to see objects in their real sizes irrespective of distance should be of advantage to the drivers of motor vehicles. The amount of this tendency may be altered by the action of drugs and by the presence of mist and fog, and this change may affect driving. One case has been reported of its change in a neurotic condition.

The psychology of vision can no longer be treated as if vision were a function of the eye alone. The foundations of a psychology of vision were firmly laid by the work of Helmholtz and his contemporaries, who made a scientific study of the sensory physiology of the eye. The most fruitful field of research at present is in those wider problems of visual perception in which the eye and the higher centres co-operate.

\section{Physiology of the Plant Cell}

P ROF. W. STILES, in his presidential address to Section K (Botany), defines the general physiology of the plant cell as those vital activities of plants which are manifested by every living cell, and are thus distinguished from those special processes such as photosynthesis, which are restricted to certain specialized organs. These general activities of living matter are respiration and the absorption and excretion of water and dissolved substances.

The usually accepted meaning of respiration is that it provides the energy for plant movements and the building up from the products of photosynthesis of substances of higher energy content than these products. Yet as respiration is a constant property of living matter, even when there is no movement and no formation of fresh material it may be questioned whether this current view of respiration is complete. As regards the synthesis of proteins and other complex substances, while there is evidence of the linkage of anabolic processes with the breaking down of carbohydrate, this anabolism only concerns the re-formation of carbohydrates, and no evidence has yet been obtained which sheds any light on the way in which energy released in respiration is transferred to the processes involved in the synthesis of proteins and other substances of higher complexity than those forming the respiratory substrate.

The absorption of water and dissolved substances by plant cells-was formerly assumed to be a simple process of diffusion through cell mem. branes, but during the present century experimental work has shown that while the absorption of non-electrolytes may sometimes take place in this way, the absorption of electrolytes is a much more complex process, for the unequal absorption of the two ions of a salt, and the entrance of the ions of a salt against their own concentration gradients are very general phenomena. Theories based on adsorption and interchange of ions have been put forward to account for these observed facts, but while such processes probably operate, it is doubtful whether they afford a complete explanation of the phenomena.

The connexion between respiration and the salt relations of tissues was pointed out in 1927, and since then further evidence has been forthcoming which has emphasized the connexion between respiration and the diffusion of salts into plant cells against the concentration gradient. The energy required for the entrance of salt thus is presumably provided by respiration, and theories have been enunciated which regard the absorption of ions as a continuous interchange of the former with the hydrogen and bicarbonate ions continuously produced within the cell as a consequence of respiration. While this interchange may be a factor in salt accumulation, there is evidence that the connexion between respiration and accumulation is generally much more indirect, and that the failure of cells to accumulate salt when respiration is lowered through deprivation of the cells of an adequate oxygen supply is related to their generally lowered vitality under this condition.

It is emphasized that salt accumulation, like respiration itself, is a vital process dependent on the protoplasm, while there is some evidence that in the absorption of water by the plant cell, there is in some, though not all, cases an active secretion of water. It is thus clear that a further analysis of the protoplasmic system is necessary for a better understanding of general cell physiology.

The general physiology of the cell is not only of fundamental importance for plant physiology in general, but it is of similar importance for all ecological investigation which is not merely descriptive, while it also impinges on the important fields of mycology and cyto-genetics. Cell physiology is the scientific basis of many important plant industries, including those of food preservation and storage.

\section{Administration of Public Education}

IN his presidential address to Section $\mathrm{L}$ (Education) Mr. John Sargent discusses the part which administration, and local administration in particular, ought to play as a medium through which the basic principles of educational science, as revealed either by a priori reasoning or as the 